\title{
Severe Cardiotoxicity in a Patient with Colorectal Cancer Treated with Bevacizumab
}

\author{
JIAN CHEN ${ }^{1,2}$, FENGCAI DU ${ }^{3}$, BAOHONG HU ${ }^{1}$, CHENG CHI ${ }^{1}$, HONGJIN CHU ${ }^{2}$, \\ LIXIN JIANG ${ }^{4}$, PENG LI ${ }^{1}$ and ZHAOHUA GONG ${ }^{1}$ \\ ${ }^{1}$ Department of Oncology, ${ }^{2}$ The Central Laboratory, and ${ }^{4}$ Department of Gastrointestinal Surgery, \\ the Affiliated Yantai Yuhuangding Hospital of Qingdao University, Yantai, P.R. China; \\ ${ }^{3}$ The First Clinical College of DaLian Medical University, Dalian, P.R. China
}

\begin{abstract}
Background/Aim: Bevacizumab combined with standard chemotherapeutics has become a choice of treatment for several kinds of cancers. Hypertension, third-degree albuminuria, thrombosis and cardiotoxicity are the reported side-effects of bevacizumab. Among them, cardiotoxicity is a most severe, but rare outcome. We report a case of a 62-yearold female with colorectal carcinoma who was given bevacizumab-containing chemotherapy for more than 20 months and achieved a stable disease during the entire course of treatment. Thereafter, she developed cardiotoxicity including grade 3 hypertension, tricuspid regurgitation, pulmonary hypertension, left ventricular diastolic dysfunction and pericardial effusion, and was discontinued from the regimen with bevacizumab. Conclusion: Although clinicallyeffective, the severe cardiotoxicity of bevacizumab developed after over 20 courses of treatment prompted us to look for optimal chemotherapy prescription in order to achieve a better clinical outcome.
\end{abstract}

Bevacizumab, a recombinant humanized monoclonal antibody against vascular endothelial growth factor (VEGF), with molecular weight of $149 \times 10^{3}$, comprises of $93 \%$ domains from human and $7 \%$ from murine sources (1). Inhibition of VEGF, an initiator of tumor angiogenesis, inhibits tumor growth and invasion (2). Tumor cells secrete VEGF to induce proliferation, mitosis of endothelial cells and promote angiogenesis (3). Overexpression of VEGF in malignant tissue is closely related to tumor cell proliferation, differentiation, metastasis and poor prognosis of patients (4). Neo-vascularization as essential for tumor cell proliferation

Correspondence to: Dr. Zhaohua Gong, Department of Oncology, the Affiliated YantaiYuhuangding Hospital of Qingdao University, 20 Yuhuangding East Road, Yantai, Shandong Provence, 264000, P.R. China. Tel: +86 18660086779, e-mail: gongzhoahuayt@163.com

Key Words: Bevacizumab, cardiotoxicity, colorectal cancer. was first proposed by Folkman in 1971 (5). Malignant neoplasms with diameter less than $2 \mathrm{~mm}$ acquires nutrition via passive diffusion, but for further proliferation, division of tumor cells requires angiogenesis $(6,7)$, which researchers believe is the key for bevacizumab targeting VEGF and exerts an anticancerous effect (8).

The Federal Drug Administration first authorized bevacizumab, combined with standard chemotherapeutics, as the first choice of treatment for metastatic colorectal cancer in 2004 (9). It was then certified as an optional drug for lung cancer, renal carcinoma and glioblastoma (10). Although obvious anticancer efficiency has been generally accepted, side-effects were also noteworthy, for example, grade 3 or hypertension (3-16\%), third-degree albuminuria (1\%-2\%), thrombosis (3\%) and cardiotoxicity (11). Recently, clinical data suggested that cardiotoxicity may be the most serious side-effect of bevacizumab, including hypertension, congestive heart failure (CHF) and left ventricular ejection fraction (LVEF) in animal models (12). Hypertension occurs most frequently, with $22.4 \%$ incidence rate in patients (13). Miller et al. carried out a phase III clinical trial, ECOG 2100 (ClinicalTrials.gov number, NCT00028990), aimed at exploring differences of clinical efficiency and safety between a single treatment group (paclitaxel, $\mathrm{n}=36$ ) and a combined treatment group (paclitaxel plus bevacizumab, $n=365$ ). This showed that the incidence of grade 3 or more hypertension and left ventricular dysfunction was $0 \%$ and $0.3 \%$ respectively in the single treatment group, but $14.8 \%$ and $0.3 \%$ respectively in the combined-treatment group $(p<0.01)(12)$. Chino et al. reported a case of a 55-year-old man with stage IV lung adenocarcinoma who received carboplatin-paclitaxelbevacizumab as second-line therapy. After four cycles of chemotherapy, he experienced syncope with a decrease in blood pressure. Electrocardiography (ECG) revealed atrial fibrillation. Cardiac ultrasonography showed a markedly reduced ejection fraction (45\%), with moderate decrease in comparison to that before chemotherapy (66\%). Bisoprolol fumarate was initiated, and the conversion to sinus rhythm was 


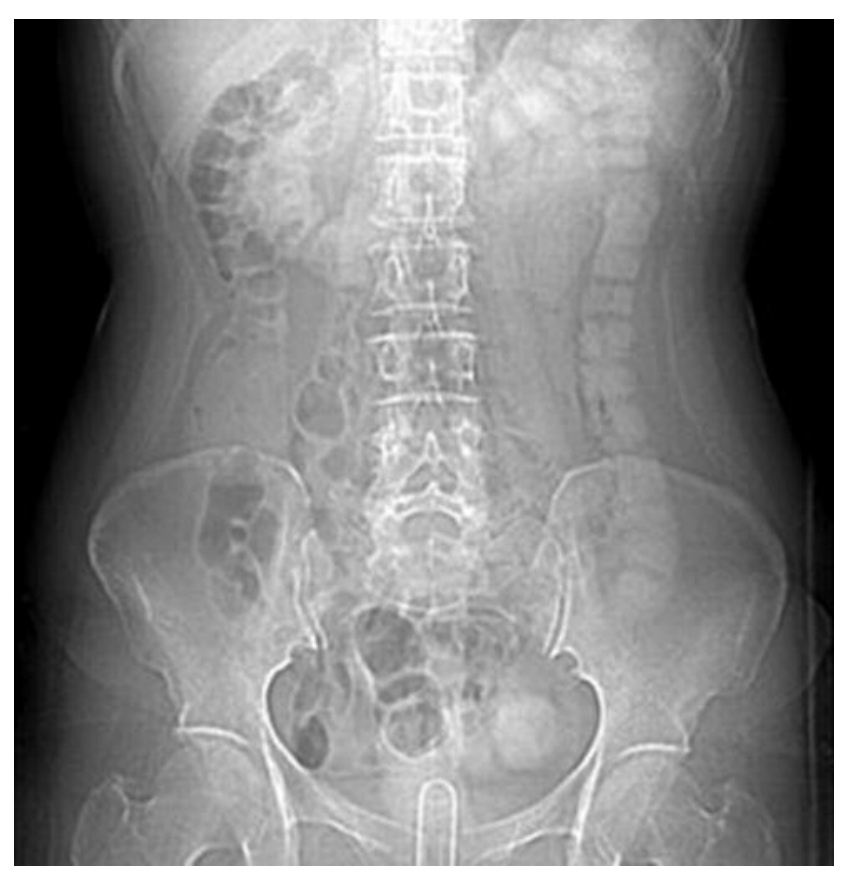

Figure 1. Abdominal computed tomography (CT) showing a neoplasm in the ascending colon with peripheral lymphadenectasis and multiple low-density lesions in the liver.

detected by ECG 4 days after the syncope, but no improvement in the ejection fraction was detected. Bevacizumab-associated cardiotoxicity was suspected, and bevacizumab maintenance therapy was discontinued. Two months after bevacizumab cessation, the ejection fraction improved to the pretreatment level (62\%) (13). Although cardiotoxicity of bevacizumab has been seen in many clinical studies, no cases of discontinued treatment or death have resulted from it as far as we are aware. Moreover, the conclusions of all clinical studies is that bevacizumab is efficient and safe. In this article, we reported a patient without history of cardiovascular and cerebrovascular diseases who accepted bevacizumab for 20 months but subsequently suffered from severe cardiotoxicity and therefore treatment was discontinued.

\section{Case Report}

A 62-year-old female was hospitalized in the Yantai Yuhuangding Hospital, Affiliated Hospital of Medical College of Qingdao University because of stomach ache on April 4, 2014. Abdominal computed tomographic (CT) scan revealed a neoplasm in the ascending colon with peripheral lymphadenectasis and several low-density lesions in the liver (Figure 1). A number of tubercles were notable in the right lung on chest CT (Figure 2). Colonoscopy showed a mass and narrowing of the colon (Figure 3). Biopsy of the

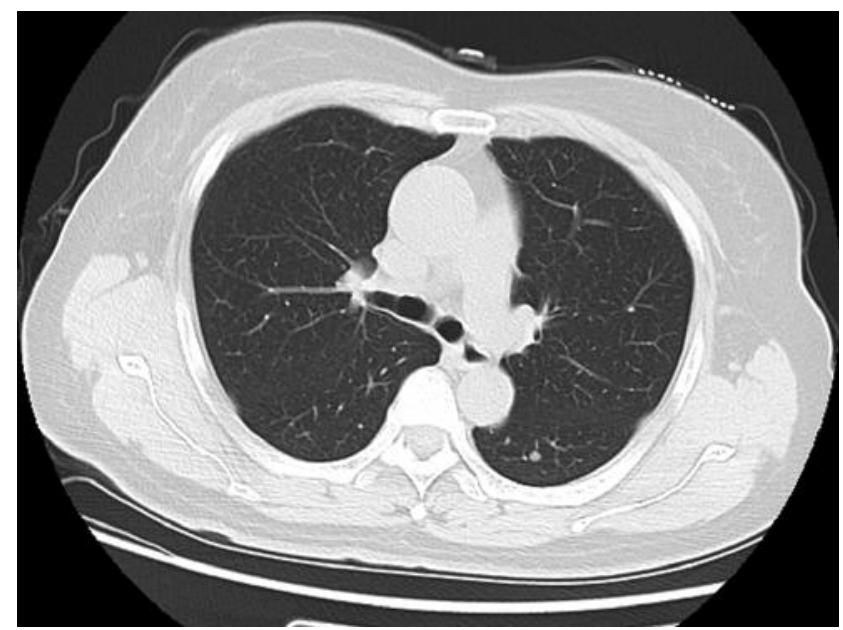

Figure 2. Chest computed tomography (CT) showing a number of tubercles in the right lung.

neoplasm in the ascending colon revealed intraepithelial neoplasia with some cancerous features (Figure 4).

We administered chemotherapy to the patient as the firstline treatment, including four courses of chemotherapy (oxaliplatin and S-1) combined with bevacizumab $(7.5 \mathrm{mg} / \mathrm{kg}$ ) from April 8, 2014 to July 15, 2014. CT scan showed partial remission by the end of the first four cycles of regimen. Later, another 17 courses of maintenance treatment (bevacizumab and S-1) were given from August 4, 2014 to November 9, 2015, after which assessment showed stable disease.

After a month, the patient was re-admitted complaining of wheezing, dizziness, and edema of the legs, with no other apparent internal injuries. Her blood pressure was 200/ $130 \mathrm{mmHg}$ with a heart rate of $116 / \mathrm{min}$. Administration of Norvasc $(5 \mathrm{mg} / \mathrm{d})$ failed to normalize blood pressure (150160/90-110 mmHg). Her ECG result indicated a sinus tachycardia. Color Doppler echocardiography (Figure 5) revealed tricuspid regurgitation, pulmonary hypertension, left ventricular diastolic dysfunction and pericardial effusion. Other laboratory results included $38 \mathrm{U} / 1$ (13-35 U/l) of aspartate aminotransferase (AST), $154 \mathrm{U} / \mathrm{L}$ (26-192 U/l) of creatinine kinase (CK), $4.96 \mathrm{ng} / \mathrm{ml}(0-2.88 \mathrm{ng} / \mathrm{ml})$ creatinine kinase-MB (CK-MB) and 511 U/l (81-234 U/l) lactate dehydrogenase (LDH). Analysis performed on the patient's urine sample suggested positivity for urine protein and occult blood. Thoracic puncture was carried out due to a large area of hydrothorax and atelectasis shown on chest CT (Figure 6). Laboratory tests showed that the pleural fluid contained no cancer cells or acid-fast bacilli (Figure 7). The hydrothorax reached approximately 19,660 $\mathrm{ml}$ in total from December 16, 2015 to February 25, 2016. The patient was forced to withdraw from the original regimen due to severe cardiotoxicity. 


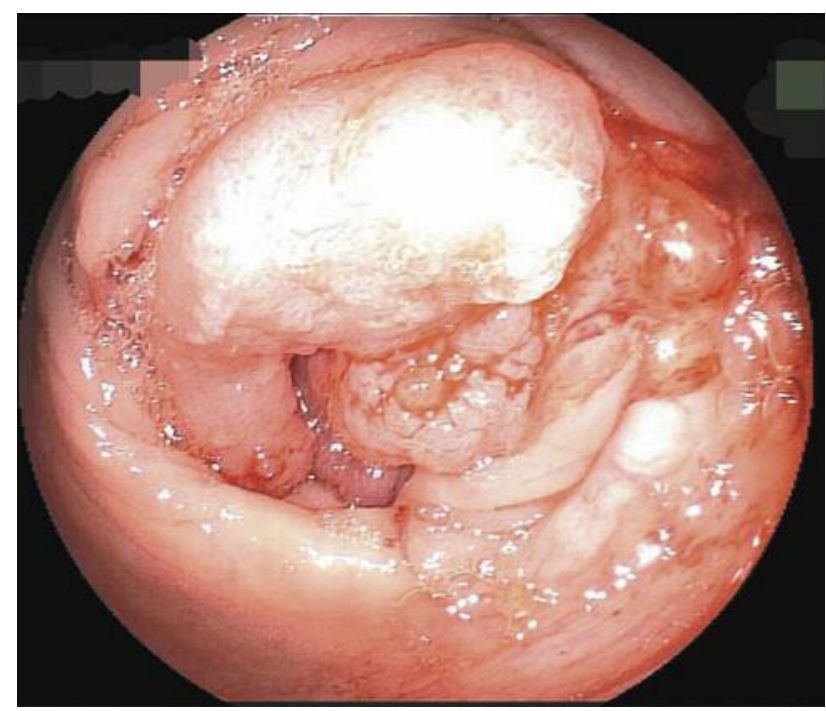

Figure 3. Colonoscopy revealed a mass and narrowing of the colon.



Figure 4. Biopsy of neoplasm in the ascending colon: intraepithelial neoplasia with part of canceration. Hematoxylin and eosin staining; magnification, $\times 100$.

Informed consent had been signed by the patient and her family.

\section{Discussion}

Bevacizumab has proved efficient for different kinds of tumors, especially colorectal cancer (8). This patient obtained obvious antitumor efficacy during the entire course of bevacizumab. The patient maintained progression-free survival for 20 months without recurrence or severe side-effects.

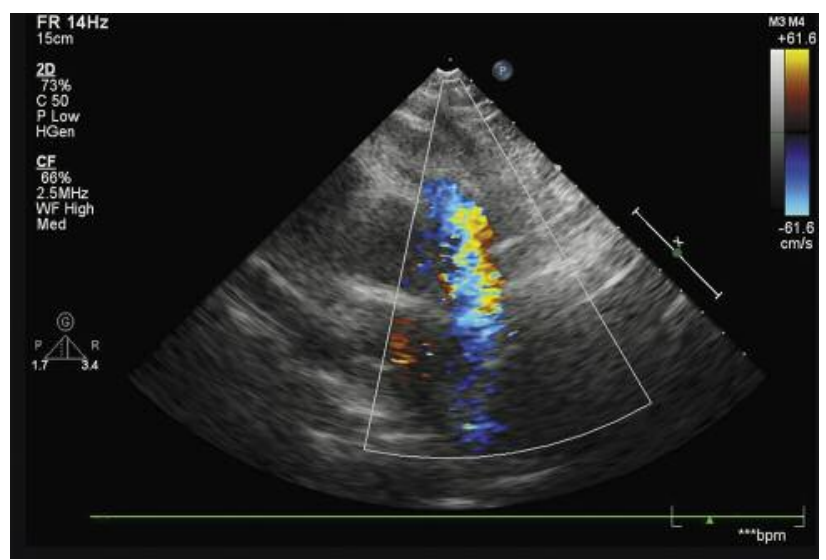

Figure 5. Echocardiography revealed tricuspid regurgitation, pulmonary hypertension, left ventricular diastolic dysfunction and pericardial effusion.

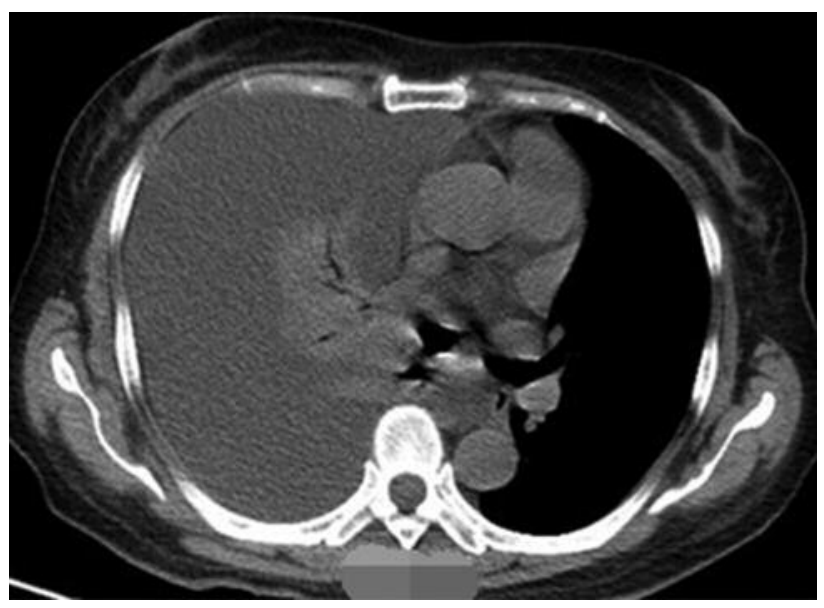

Figure 6. Chest computed tomography (CT) showing a large area of hydrothorax.

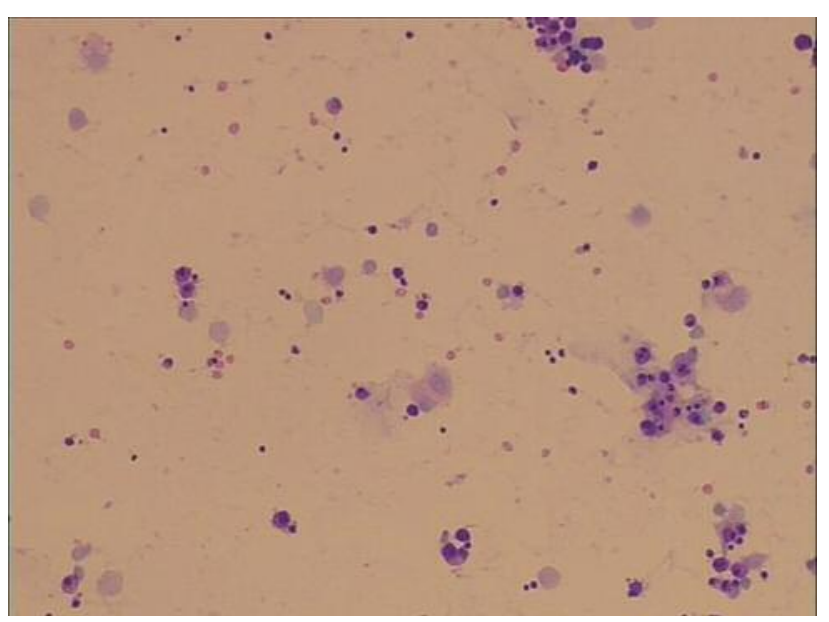

Figure 7. Test of pleural fluid revealed a transudate without cancer cells and acid-fast bacilli. Hematoxylin and eosin staining; magnification, $\times 100$. 
Although the anticancer efficiency of bevacizumab has been proven, its side-effects are also noteworthy, such as hypertension, albuminuria, thrombosis and cardiotoxicity (11). Cardiotoxicity may be the most serious side-effect for bevacizumab, including hypertension, CHF and LVEF. Hypertension is usually the first sign of toxicity. The patient mentioned in this report is a representative case with severe cardiotoxicity related to the use of bevacizumab. The patient complained of wheezing, dizziness, and edema of the legs with grade 3 hypertension. ECG tests showed sinus tachycardia. Color Doppler echocardiography revealed tricuspid regurgitation, pulmonary hypertension, left ventricular diastolic dysfunction and pericardial effusion, and AST, CK, CK-MB and LDH were abnormally high; all of which revealed disruption of normal cardiac function. Positive urine protein and large quantities of hydrothorax with no cancerous cells seen can prove these abnormalities resulted from bevacizumab based on previously published research (14). In fact, the molecular mechanism of cardiotoxicity caused by bevacizumab is not conclusive.

Generally, differently from anthracyclines, anti-angiogenesis drugs often cause reversible cardiac damage by either a 'target effect' or 'missing target effect' (15). The former hypothesis means that bevacizumab inhibits VEGF which is indispensable for cardiac function. Firstly, VEGF induces diastole of endothelium-dependent coronary artery by stimulating endothelial cells to release nitrous oxide and prostacyclin, as a result, it can relieve hypertension. Bevacizumab leads to vasoconstriction and hypertension by inhibiting VEGF. Secondly, inhibition by VEGF of the kidney can induce occurrence of renal thrombotic microangiopathy and aggravation of hypertension (16). Lastly, it has also been noted that VEGF was overexpressed in patients with myocardial infarction (17) or cardiac pressure overload (18). This was believed to be a cardiac compensatory mechanism. Animal experiments by Izumiya et al. demonstrated that VEGF can reduce cardiac hypertrophy caused by overload (19). Therefore, VEGF is crucial for normal cardiac function and its inhibition by bevacizumab can lead to cardiotoxicity. Our case confirms that bevacizumab affects the important roles of VEGF in heart function. For example, inhibition of the AMP-activated protein kinase, platelet-derived growth factor receptors and VEGFR signaling pathway, which involves cardiac energy metabolism regulation, results in cardiac dysfunction, including reduced LVEF and CHF $(20,21)$.

Moreover, another question arises from our case: How long does the latency of severe cardiotoxicity last before manifesting syndromes? In our case it was 20 months after the first bevacizumab administration. In addition to the unclear mechanism of cardiotoxicity caused by bevacizumab, an optimal time course for patients using bevacizumab needs to be further evaluated in order to achieve the most advantageous clinical efficacy.

\section{Acknowledgements}

This work was supported by the National Natural Science Foundation of China (81071758); Shandong Sci-nence and Technology Development Project (2015GSF118142); Shandong Medicine and Health Science Technology Program (2016WS0706); China International Medical Foundation (Z20140615325); Natural Science Foundation of Shandong Province Joint Programme (ZR2016HL26, ZR2015HL069); and YantaiYuhuangding Hospital Initiative Foun $\neg$ dation for Young Scientist $(201526,201402)$; Yantai Science and Technology Program (2016WS013, 2015WS018, 1605350099).

\section{References}

1 Hara M, Nagasaki T, Shiga K and Takeyama H: Suppression of cancer-associated fibroblasts and endothelial cells by itraconazole in bevacizumab-resistant gastrointestinal cancer. Anticancer Res 36(1): 169-177, 2016.

2 Lien S and Lowman HB: Therapeutic anti-VEGF antibodies. Handb Exp Pharmacol 181: 131-50, 2008.

3 Lee JH, Canny MD, De Erkenez A, Krilleke D, Ng YS, Shima DT, Pardi A and Jucker F: A therapeutic aptamer inhibits angiogenesis by specifically targeting the heparin binding domain of VEGF165. Proc Natl Acad Sci USA 102(52): 1890218907, 2005.

4 Lin YS, Nguyen C, Mendoza JL, Escandon E, Fei D, Meng YG and Modi NB: Preclinical pharmacokinetics, interspecies scaling and tissue distribution of a humanized monoclonal antibody against vascular endothelial growth factor. J Pharmacol Exp Ther 288(1): 371-378, 1999.

5 Paiva TF Jr., de Jesus VH, Marques RA, da Costa AA, de Macedo MP, Peresi PM, Damascena A, Rossi BM, Begnami MD and de Lima VC: Angiogenesis-related protein expression in bevacizumab-treated metastatic colorectal cancer: NOTCH1 detrimental to overall survival. BMC cancer 15: 643, 2015.

6 Ferrara N, Gerber HP and LeCouter J: The biology of VEGF and its receptors. Nat Med 9(6): 669-676, 2003.

7 Nagy JA, Vasile E, Feng D, Sundberg C, Brown LF, Manseau EJ, Dvorak AM and Dvorak HF: VEGF-A induces angiogenesis, arteriogenesis, lymphangiogenesis and vascular malformations. Cold Spring Harb Symp Quant Biol 67: 227-237, 2002.

8 Hurwitz H, Fehrenbacher L, Novotny W, Cartwright T, Hainsworth J, Heim W, Berlin J, Baron A, Griffing S, Holmgren E, Ferrara N, Fyfe G, Rogers B, Ross R and Kabbinavar F: Bevacizumab plus irinotecan, fluorouracil and leucovorin for metastatic colorectal cancer. N Engl J Med 350(23): 2335-2342, 2004.

9 Escudier B, Pluzanska A, Koralewski P, Ravaud A, Bracarda S, Szczylik C, Chevreau C, Filipek M, Melichar B, Bajetta E, Gorbunova V, Bay JO, Bodrogi I, Jagiello-Gruszfeld A and Moore N: Bevacizumab plus interferon alfa-2a for treatment of metastatic renal cell carcinoma: a randomised, double-blind phase III trial. Lancet 370(9605): 2103-2111, 2007.

10 Friedman HS, Prados MD, Wen PY, Mikkelsen T, Schiff D, Abrey LE, Yung WK, Paleologos N, Nicholas MK, Jensen R, Vredenburgh J, Huang $J$, Zheng $M$ and Cloughesy $T$ : Bevacizumab alone and in combination with irinotecan in recurrent glioblastoma. J Clin Oncol 27(28): 4733-4740, 2009.

11 Saif MW and Mehra R: Incidence and management of bevacizumab-related toxicities in colorectal cancer. Expert Opin Drug Saf 5(4): 553-566, 2006. 
12 Lin JM, Guo WX, Wang Y, Liu Y, Li YR, Li H and Zhou J: Establishment of chronic heart failure rat model of Xin-qi deficiency complicated blood stasis and edema syndrome and judgment of diagnosis information integration. Zhongguo Zhong Xi Yi Jie He Za Zhi 34(12): 1457-62, 2014.

13 Bonsa F, Gudina EK and Hajito KW: Prevalence of hypertension and associated factors in Bedele Town, Southwest Ethiopia. Ethiop J Health Sci 24(1): 21-6, 2014.

14 Miller K, Wang M, Gralow J, Dickler M, Cobleigh M, Perez EA, Shenkier T, Cella D and Davidson NE: Paclitaxel plus bevacizumab versus paclitaxel alone for metastatic breast cancer N Engl J Med 357(36): 2666-2676, 2007.

15 Chino H, Amano Y, Yamauchi Y, Matsuda J, Takeda N, Tanaka G, Takai D and Nagase T: Cardiogenic syncope possibly related to bevacizumab-containing combination chemotherapy for advanced non-small cell lung cancer. J Thorac Dis 8(9): 2646-2650, 2016.

16 Lee CS, Alwan LM, Sun X, McLean KA and Urban RR: Routine proteinuria monitoring for bevacizumab in patients with gynecologic malignancies. J Oncol Pharm Pract 22(6): 771-776, 2015.

17 Cheng $\mathrm{H}$ and Force $\mathrm{T}$ : Why do kinase inhibitors cause cardiotoxicity and what can be done about it? Prog Cardiovasc Dis 53(2): 114-120, 2010.

18 Allegra CJ, Yothers G, O'Connell MJ, Sharif S, Colangelo LH, Lopa SH, Petrelli NJ, Goldberg RM, Atkins JN, Seay TE, Fehrenbacher L, O'Reilly S, Chu L, Azar CA and Wolmark N: Initial safety report of NSABP C-08: A randomized phase III study of modified FOLFOX6 with or without bevacizumab for the adjuvant treatment of patients with stage II or III colon cancer. J Clin Oncol 27(20): 3385-3390, 2009.
19 Heba G, Krzeminski T, Porc M, Grzyb J, Ratajska A and Dembinska-Kiec A: The time course of tumor necrosis factoralpha, inducible nitric oxide synthase and vascular endothelial growth factor expression in an experimental model of chronic myocardial infarction in rats. J Vasc Res 38(3): 288-300, 2001.

20 Shyu KG, Liou JY, Wang BW, Fang WJ and Chang H: Carvedilol prevents cardiac hypertrophy and overexpression of hypoxiainducible factor-1alpha and vascular endothelial growth factor in pressure-overloaded rat heart. J Biomed Sci 12(2): 409-420, 2005.

21 Izumiya Y, Shiojima I, Sato K, Sawyer DB, Colucci WS and Walsh $\mathrm{K}$ : Vascular endothelial growth factor blockade promotes the transition from compensatory cardiac hypertrophy to failure in response to pressure overload. Hypertension 47(5): 887-893, 2006.

22 Greineder CF, Kohnstamm S and Ky B: Heart failure associated with sunitinib: lessons learned from animal models. Curr Hypertens Rep 13(6): 436-441, 2011.

23 Gupta R and Maitland ML: Sunitinib, hypertension and heart failure: a model for kinase inhibitor-mediated cardiotoxicity. Curr Hypertens Rep 13(6): 430-435, 2011.
Received April 6, 2017

Revised May 25, 2017

Accepted May 27, 2017 